\title{
Association between hand grip strength and spirometric parameters: Korean National health and Nutrition Examination Survey (KNHANES)
}

\author{
Chang Hoon Han ${ }^{1}$, Jae Ho Chung ${ }^{2}$ \\ ${ }^{1}$ Department of Internal Medicine, National Health Insurance Service Ilsan Hospital, Goyang, Republic of Korea; ${ }^{2}$ Department of Internal Medicine, \\ International St. Mary's Hospital, Catholic Kwandong University College of Medicine, Incheon, Republic of Korea \\ Contributions: (I) Conception and design: All authors; (II) Administrative support: None; (III) Provision of study materials or patients: None; \\ (IV) Collection and assembly of data: All authors; (V) Data analysis and interpretation: All authors; (VI) Manuscript writing: All authors; (VII) Final \\ approval of manuscript: All authors. \\ Correspondence to: Jae Ho Chung, MD. Department of Internal Medicine, International St. Mary's hospital, 22711 Simgokro $100 G i l 25$ Seo-gu \\ Incheon, Republic of Korea. Email: klaatu70@gmail.com.
}

\begin{abstract}
Background: We investigated the associations between hand grip strength (HGS) and spirometric parameters.

Methods: A total of 5,303 participants over 40 years of age, who underwent spirometry and HGS testing, were selected from the Korean National Health and Nutrition Examination Survey 2014-2015. Outcome measures were forced expiratory volume in $1 \mathrm{~s}\left(\mathrm{FEV}_{1}\right)$, forced vital capacity (FVC), $\mathrm{FEV}_{1} / \mathrm{FVC}$, and peak expiratory flow rate (PEFR). Unadjusted and adjusted linear regressions were used for the analyses.

Results: Mean HGS in the obstructive group was: male: $38.9 \pm 6.9 \mathrm{~kg}$; female: $24.2 \pm 4.8 \mathrm{~kg}$, which was significantly less than that in the normal group (male: $42.2 \pm 7.2 \mathrm{~kg}, \mathrm{P}<0.001$; female: $26.0 \pm 4.6 \mathrm{~kg}, \mathrm{P}<0.001$ ). In a multiple linear regression model, HGS was significantly associated with $\mathrm{FEV}_{1}$ (male: $\beta=0.18, \mathrm{P}<0.001$, female: $\beta=0.21, P<0.001$ ), FVC (male: $\beta=0.23, P<0.001$, female: $\beta=0.24, P<0.001$ ) and PEFR (male: $\beta=0.13$, $\mathrm{P}<0.001$, female: $\beta=0.14, \mathrm{P}<0.001)$ after adjustment.
\end{abstract}

Conclusions: We found associations between muscle strength as evaluated by HGS and spirometric pulmonary function parameters.

Keywords: Airway; hand grip strength (HGS); obstruction

Submitted Mar 21, 2018. Accepted for publication Sep 21, 2018.

doi: $10.21037 /$ jtd.2018.10.09

View this article at: http://dx.doi.org/10.21037/jtd.2018.10.09

\section{Introduction}

Peripheral muscle weakness is commonly observed in patients with obstructive lung disease, and its possible contribution to exercise intolerance has been reported (1). Hand grip strength (HGS), a simple bedside tool test, is portable, relatively inexpensive, and a reliable measurement of muscular strength (2). Low HGS is associated with mortality, disability, and other health-related complications among middle-aged and older people (3). It is also associated with mortality in patients with COPD (4) and is an attractive tool not only for assessing exercise capacity but also for predicting the prognosis of COPD patients in both clinical practice and a research context (5). Although handgrip dynamometry is reliably prognostic, the test is rarely used during routine assessment in pulmonary departments. A few contradictory studies on HGS measurements in patients with obstructive lung disease have appeared. Gosselink et al. (6) showed that HGS was reduced in patients with obstructive lung disease, and Puhan et al. (4), in a prospective study, found that HGS predicted mortality in patients with obstructive lung disease. In a large primary care study, patients with obstructive lung disease had a 
lower HGS than a healthy control group (7), but the HGSs of patients with moderate to severe obstructive lung disease and controls were similar in another study (6). Marino et al. (8) founded that there was no association between HGS and spirometric lung function result, as assessed by the predicted forced expiratory volume percent in $1 \mathrm{~s}$ $\left(\mathrm{FEV}_{1} \%\right)$ among moderate to severe chronic obstructive pulmonary disease (COPD). Recent studies $(9,10)$ have found that HGS is not related to lung function parameters such as forced vital capacity (FVC) or $\mathrm{FEV}_{1} \%$. However, as the evidence is inconsistent, we investigated the association between HGS and spirometric parameters by analyzing samples of adults enrolled in the sixth Korean National Health and Nutrition Examination Survey (KNHANES VI).

\section{Methods}

\section{Study populations}

This present study was using the data obtained from KNHANES VI [2014-2015]. KNHANES surveys are annually investigated for assess the health and nutritional status of the non-institutionalized populations who living in South Korea. These investigations are composed of a health interview survey, health examination survey, and nutrition survey which are conducted by well-trained interviewer. Annually, subjects are selected to represent Korean populations who are $\geq 18$ years old using a multistage clustered and stratified randomize sampling method. So, KNHANES VI represent national-wide cross-sectional surveys. Of these participants, the data from 2,412 males and 2,891 female subjects aged $\geq 40$ years who underwent spirometry and HGS were analyzed. Subjects were excluded if they were $<40$ years of age $(n=6,092)$, and if data were unavailable to evaluate HGS $(\mathrm{n}=3,529)$. After these exclusions, a total of 5,303 participants were analyzed for the present study.

\section{Socioeconomic and demographic factors}

Age, household income, and occupation were included as sociodemographic factors in the analyses. Household income was categorized into four quartiles. Education level was categorized into four groups: elementary school or lower, middle school, high school, and college school or higher. Occupational status was divided into employed and unemployed (including housewife and student).
Individuals who had smoked five packs (100 cigarettes) or more over their lifetime, and who currently smoked, were classified as "smokers," whereas less than 100 cigarettes was considered "former-smokers" and "non-smokers" (11). Participants were classified as "risky drinkers" if they answered 12 or more heavy drinking episodes (consumption of five or more alcoholic beverages in a single day) during the last year (12). By the American College of Sports Medicine Guidelines (13), regular exercise was defined. Diabetes mellitus (DM), angina, or myocardial infarction, and stroke were determined by a self-administered questionnaire, as follows: "Have you ever been diagnosed with DM/angina or myocardial infarction/stroke by a doctor? Yes or no?" Energy and nutrients intake were assessed using the $24 \mathrm{~h}$ recall method, which provides valid and reliable information (14). Intakes of total fat, protein, energy, and carbohydrate were calculated from the food items consumed.

\section{Spirometry and definition of airflow obstruction}

Well-trained technicians measured FVC, $\mathrm{FEV}_{1}$, the $\mathrm{FEV}_{1}$ / FVC ratio, peak expiratory flow rate (PEFR), and maximal mid-expiratory flow (MMEF) using spirometer (model 2130; SensorMedics, CA, USA). The quality-control programs were conducted; the spirometer calibration was done by technicians before conducting spirometry every morning using the American Thoracic Society/European Respiratory Society criteria of repeatability and acceptability tests. All spirometry values were prebronchodilator results. Normal predicted values were derived from large population studies of healthy subjects. This variable used to predict normal values included sex, age, ethnicity, height and weight (15). Spirometry results were classified as a normal, obstructive, or restrictive pattern as the American Thoracic Society criteria. Subjects with $\mathrm{FEV}_{1} / \mathrm{FVC}$ $\geq 70 \%$ and $\mathrm{FVC} \geq 80 \%$ of the normal predicted value were classified as normal (16). $\mathrm{FEV}_{1} / \mathrm{FVC}<70 \%$ were classified as obstructive pattern. When $\mathrm{FEV}_{1} / \mathrm{FVC} \geq 70 \%$ and $\mathrm{FVC}$ was $<80 \%$ of the normal predicted value, the participants were classified as the restrictive pattern.

\section{HGS}

HGS was performed using a digital hand dynamometer (Digital grip strength dynamometer, T.K.K 5401, Takei Scientific Instruments Co., Ltd., Tokyo, Japan). Grip strength was measured in a standing position and performed 
the maximum grip strength three times with the dominant hands (17). At least a $30 \mathrm{~s}$ rest interval was allowed between each three HGS measurement (18).

\section{Ethical issues}

The Institutional Review Board of the Korea Centers for Disease Control and Prevention approved this study protocol (nos. 2014-12EXP-03-5C, 2015-01CON-02-6C), and all participants signed informed consent forms.

\section{Data analyses}

All sampling and weight variables used in this our study were stratified, and the SPSS (SPSS Inc., Chicago, IL, USA) was used for all statistical analyses to ensure appropriate estimates and standard errors. All clinical characteristics were compared using the chi-square test or Fisher's exact test for categorical variables and analysis of variance for each variable.
We made multiple adjustments for the following potential confounders of the association between HGS and spirometric parameters outcomes: age, weight, height, family income, education level, employment status, physical activity level, tobacco and alcohol use, presence of co-morbidities (self-reported DM, angina or myocardial infarction, and stroke), and nutritional factors. Spearman correlation coefficients were used to examine the relationships between $\mathrm{HGS}$ and $\mathrm{FEV}_{1}, \mathrm{FVC}, \mathrm{FEV}_{6}, \mathrm{MMEF}$, and PEFR. Variables significantly associated with HGS and spirometry parameters were used in multivariate analyses. The level of statistical significance for each test was $\mathrm{P}<0.05$.

\section{Results}

\section{Baseline characteristics}

The baseline characteristics of our study population are shown in Table 1. According to their pulmonary function test results, subjects were categorized into three groups (normal: $76.1 \%$, restrictive: $8.8 \%$, or obstructive: $15.1 \%$ ).

Table 1 Clinical characteristics of study participants

\begin{tabular}{|c|c|c|c|c|c|c|c|c|c|c|}
\hline \multirow{2}{*}{$\begin{array}{l}\text { Characters } \\
\text { Age (years) }\end{array}$} & \multicolumn{5}{|c|}{ Male } & \multicolumn{5}{|c|}{ Female } \\
\hline & $\begin{array}{c}\begin{array}{c}\text { Obstructive } \\
(n=608)\end{array} \\
64.9 \pm 9.1\end{array}$ & $\begin{array}{c}\begin{array}{c}\text { Restrictive } \\
(\mathrm{n}=242)\end{array} \\
60.7 \pm 10.5\end{array}$ & $\begin{array}{c}\begin{array}{c}\text { Normal } \\
(n=1,562)\end{array} \\
55.0 \pm 10.1\end{array}$ & $<0.001$ & $a, b, c$ & $\begin{array}{c}\begin{array}{c}\text { Obstructive } \\
(n=195)\end{array} \\
65.4 \pm 9.1\end{array}$ & $\begin{array}{c}\begin{array}{c}\text { Restrictive } \\
(n=223)\end{array} \\
60.5 \pm 9.5\end{array}$ & $\begin{array}{c}\begin{array}{c}\text { Normal } \\
(n=2,473)\end{array} \\
56.1 \pm 10.3\end{array}$ & $<0.001$ & $a, b, c$ \\
\hline Height, m & $1.67 \pm 0.06$ & $1.68 \pm 0.06$ & $1.69 \pm 0.06$ & $<0.001$ & $a, b$ & $1.55 \pm 0.06$ & $1.55 \pm 0.05$ & $1.56 \pm 0.06$ & $<0.001$ & $a, b$ \\
\hline Body mass index, $\mathrm{kg} / \mathrm{m}^{2}$ & $24.1 \pm 2.7$ & $25.9 \pm 3.6$ & $24.3 \pm 2.9$ & $<0.001$ & $b, c$ & $23.6 \pm 2.9$ & $25.4 \pm 3.8$ & $23.8 \pm 3.1$ & $<0.001$ & $b, c$ \\
\hline Smoking status & & & & 0.003 & $a, c$ & & & & 0.185 & \\
\hline Never & $104(17.1)$ & $58(24.0)$ & $333(21.3)$ & & & 182 (93.3) & $210(94.2)$ & $2,292(92.7)$ & & \\
\hline Ex-former & $3(0.5)$ & $5(2.1)$ & $36(2.3)$ & & & $0(0)$ & $4(1.8)$ & $20(0.8)$ & & \\
\hline Family income & & & & $<0.001$ & $a, b$ & & & & $<0.001$ & $a, b, c$ \\
\hline Low & $165(27.1)$ & $47(19.4)$ & $206(13.2)$ & & & $75(38.5)$ & $49(22.0)$ & $462(18.7)$ & & \\
\hline Moderate-low & $195(32.1)$ & $72(29.8)$ & $366(23.4)$ & & & $46(23.6)$ & $71(31.8)$ & $622(25.2)$ & & \\
\hline Moderate-high & $132(21.7)$ & $60(24.8)$ & $436(27.9)$ & & & 32 (16.4) & 48 (21.5) & $643(26.0)$ & & \\
\hline High & $126(20.7)$ & $63(26.0)$ & $554(35.5)$ & & & $42(21.5)$ & $55(24.7)$ & 746 (30.2) & & \\
\hline
\end{tabular}

Table 1 (continued) 
Table 1 (continued)

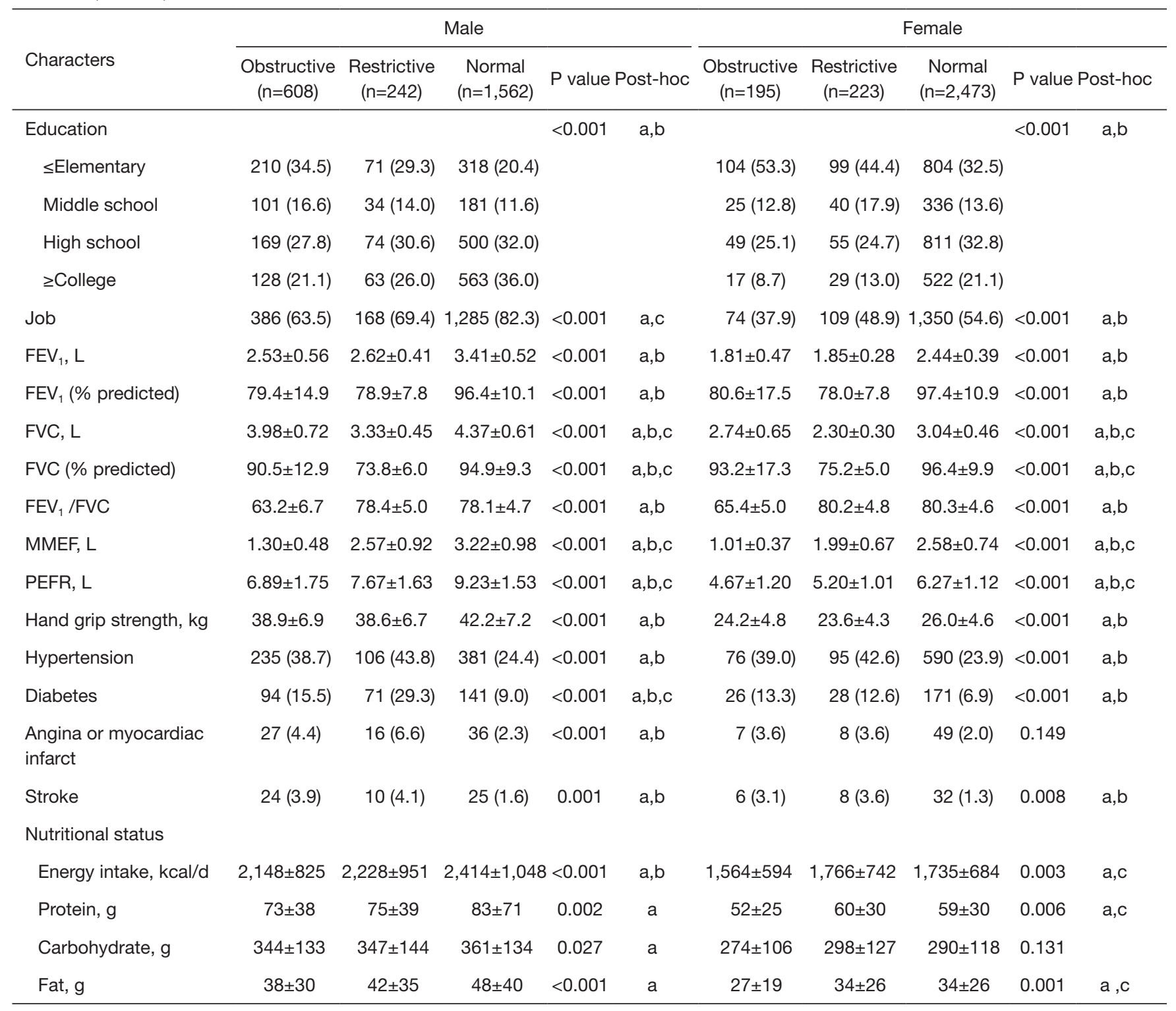

Values are presented as numbers (\%) or means \pm SD. a, normal vs. obstructive; b, normal vs. restrictive; c, obstructive vs. restrictive. FEV , forced expiration volume after $1 \mathrm{~s}$; FVC, forced vital capacity; MMEF, Maximal mid-expiratory flow; PEFR, peak expiratory flow rate.

The difference between males and females were $64.8 \%$ vs. $85.5 \%, 25.2 \%$ vs. $6.7 \%$, and $10.0 \%$ vs. $7.7 \%$ for normal, obstructive, and restrictive lung function, respectively. Significant differences were observed among the three groups in age $(\mathrm{P}<0.001$, both), weight $(\mathrm{P}<0.001$, both $)$, height $(\mathrm{P}<0.001$, both), and body mass index (BMI; $\mathrm{P}<0.001$, both) in males and females. The obstructive group showed older age and had lower BMI and body weight than those of the other groups. The restrictive group showed higher body weight and BMI than those of the other groups. There was significant differences in family income $(\mathrm{P}<0.001$, both $)$ and education level $(\mathrm{P}<0.001$, both $)$ were observed in males and females, respectively. The obstructive group showed lower family income and education level than the other groups. $\mathrm{DM}(\mathrm{P}<0.001$, both $)$ and hypertension $(\mathrm{P}<0.001)$ were significantly more frequently observed in the restrictive group (males and females) than those in the other groups. 
Table 2 Association of hand grip strength and lung function

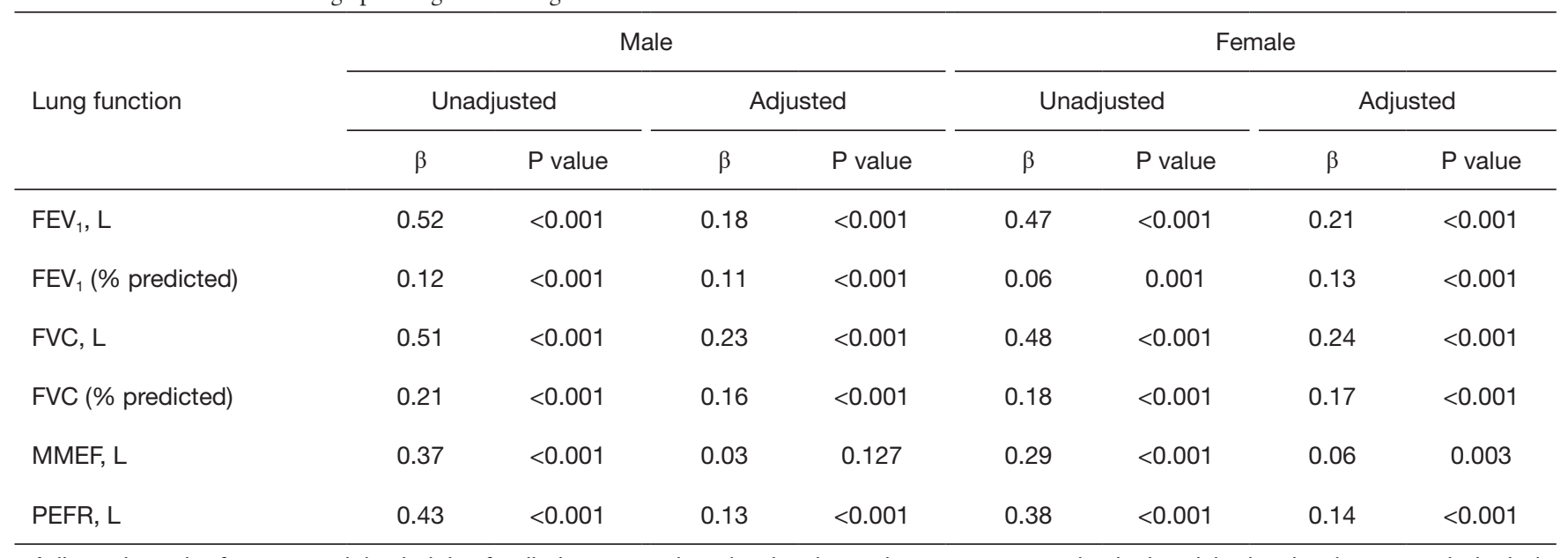

Adjusted results for age, weight, height, family income, education level, employment status, physical activity level, tobacco and alcohol use, presence of co-morbidities (self-reported diabetes mellitus, angina or myocardiac infarct and stroke) and nutritional factors. $\beta$, beta coefficient; $F E V_{1}$, forced expiratory volume in 1 second; FVC, forced vital capacity; MMEF, Maximal mid-expiratory flow; PEFR, peak expiratory flow rate.

\section{Association between HGS and lung function parameters}

The respiratory parameters $\left(\mathrm{FVC}, \mathrm{FEV}_{1}, \mathrm{MMEF}\right.$, and PEFR) were significantly lower in the obstructive group, compared to the control population $(\mathrm{P}<0.001)$. Mean HGS in the male obstructive group was $38.9 \pm 6.9 \mathrm{~kg}$ and was significantly less than that of normal males $(42.2 \pm 7.2 \mathrm{~kg}$, $\mathrm{P}<0.001)$. In females, mean HGS in the obstructive group was $24.2 \pm 4.8 \mathrm{~kg}$ and was significantly less than that in the normal controls $(26.0 \pm 4.6 \mathrm{~kg}, \mathrm{P}<0.001)$. Table 2 shows the associations between HGS and lung function parameters. $\operatorname{FEV}_{1}(\beta=0.52, \mathrm{P}<0.001)$, FVC $(\beta=0.51, \mathrm{P}<0.001)$, and PEFR $(\beta=0.43, \mathrm{P}<0.001)$ were positively correlated with muscle strength in the unadjusted male model, and $\mathrm{FEV}_{1}$ $(\beta=0.47, \mathrm{P}<0.001)$, FVC $(\beta=0.48, \mathrm{P}<0.001)$, and PEFR $(\beta=0.38, \mathrm{P}<0.001)$ were positively correlated with HGS in the unadjusted female model. In a multiple linear regression model, HGS was significantly associated with $\mathrm{FEV}_{1}$ (male: $\beta=0.18, P<0.001$, female: $\beta=0.21, P<0.001), F V_{1 \%}$ of predicted value (male: $\beta=0.11, \mathrm{P}<0.001$, female: $\beta=0.13$, $\mathrm{P}<0.001$ ), FVC (male: $\beta=0.23, \mathrm{P}<0.001$, female: $\beta=0.24$, $\mathrm{P}<0.001$ ), FVC\% of predicted value (male: $\beta=0.16, \mathrm{P}<0.001$, female: $\beta=0.17, P<0.001$ ), and PEFR (male: $\beta=0.13$, $\mathrm{P}<0.001$, female: $\beta=0.14, \mathrm{P}<0.001$ ) when adjusted for age, weight, height, family income, education level, employment status, regular exercise, tobacco and alcohol use, presence of co-morbidities (self-reported DM, angina or myocardial infarction and stroke), and nutritional factors.

\section{Discussion}

In this representative sample of South Koreans who aged $\geq 40$ years, we found associations between muscle strength, as evaluated by HGS and spirometric pulmonary function parameters. In further analyses, HGS was associated with pulmonary disease severity, assessed as $\mathrm{FEV}_{1} \%$ of the predicted value independent of age, height, and smoking habit.

We found that reduced muscle strength was associated with reduced pulmonary function. This association was found between HGS and FVC, FEV ${ }_{1}$, and MMEF (female only) in our study. The pathogenesis of the association between HGS and pulmonary function is not well established. The finding that HGS strongly predicts the development of functional disabilities and mortality (19), systemic factors (e.g., systemic inflammation and hormonal changes due to the aging process), and musculature changes that affect both pulmonary function and muscle strength, may manifest most clearly in a decline in HGS in the general population (20). HGS is a simple measure of upper limb muscle function and is associated with mortality in patients with COPD (4); HGS testing is an attractive option not only when assessing exercise capacity but also when seeking to predict COPD prognosis both in clinical practice and the research context (5). Although there was an association between HGS and COPD mortality, there was no association between HGS and risk for hospital 
admission for COPD patients (21). HGS is associated with upper and lower limb muscle strength among patients with moderate to severe COPD. The interrelationships between pulmonary functions and HGS in previous studies had shown different results; some studies have shown no different HGS among subjects with and without obstructive lung disease $(1,6,22)$, whereas others have shown lower HGS in patients with obstructive lung disease (7). A recent population-based study in Sweden reported no differences in HGS when comparing subjects with and without obstructive lung disease, whereas those with obstructive lung disease and heart disease such as angina or myocardiac infarct patients had lower HGS than those without heart disease (23). These contradictory findings may be due to enrolling patients with different severities of obstructive lung disease. Severe obstructive lung disease subjects, GOLD 3-4 grade, had significantly lower HGS, compared to subjects with non-obstructive lung disease. But, each of the GOLD grades includes a wider range of FEV1\% of predicted value, which may, as a continuous variable, be more sensitive when evaluating HGS in relation to obstructive lung disease severity. Actually, a decrease in the FEV1\% predicted value have shown association between lower HGS in obstructive lung disease, which persisted after adjusting for confounders. Kutsuzawa et al. (24) measured reduced HGS (mean $70 \%$ of predictive value) in patients with obstructive lung disease and a similar association between airflow obstruction severity and HGS. Reduced HGS (65\% of predictive value) was observed in obstructive lung disease patients and a more severe airflow obstruction (25). Bernard et al. (26) showed a significant correlation between $\mathrm{FEV}_{1} \%$ of predictive value and upper arm quadriceps muscle strength. This discrepancy probably resulted from differences in skeletal muscles under investigation and muscle strength measurement methods. Taken together, there is an apparent relationship between muscle strength and airflow obstructive disease severity, as assessed by the $\mathrm{FEV}_{1} \%$ of predicted value, independent of age, height, and smoking habit in patients with obstructive lung disease. In this present study, we also showed airflow obstruction was correlated with HGS. Recent studies $(9,10)$ that used data from the same KHNANES reported different results. Jeong et al. (9) investigated 421 patients with COPD diagnosed using spirometric lung function tests, and 2,542 controls, and found that HGS was not related to forced vital capacity $(\beta=0.04, \mathrm{P}=0.70)$ or $\mathrm{FEV}_{1} \%(\beta=0.11, \mathrm{P}=0.24)$ in multivariable analyses. Lee et al. (10) showed that lung function was not significantly associated with HGS
(832 non-COPD subjects and 832 COPD patients were evaluated after propensity score matching). We included all of the 5,303 subjects who underwent spirometry and HGS; our subject numbers were thus larger than those of the two cited studies. This may explain why the studies differ; a future, well-designed prospective study is required.

Our study also showed that HGS was associated with PEFR. PEFR is a relatively inexpensive measurement such as FEV1, and was used in previous studies to evaluate HGS, muscle strength, and lung function (27). PEFR represents larger airways airflow and represent effort dependent maximal expiratory flow (28). Fragoso et al. (29) reported that PEFR is associated with a reduced ability to participate in the activities of daily life and increased disability and death, independent of multiple potential confounders, such as age, smoking status, and presence of chronic lung disease, such as COPD. In addition, Sillanpää et al. (30) showed that decreased mobility is directly regulated by a decline in muscle strength and muscle power and is also partly mediated by decreases in pulmonary function. The reasons for the association between HGS and PEFR are obscure; however, HGS has been used as a sarcopenia indicator, providing information regarding the amount of muscle mass (31), and PEFR is influenced by respiratory muscle strength (32). Thus, reductions in respiratory muscle mass are likely to decrease PEFR performance.

The present study had some limitations. First, the crosssectional study model can't explain cause-effect relationships; such a study design may result in over-generalization of the findings. Our results can be generalized only to ethnic Koreans. Second, post-bronchodilator spirometry measurement was not evaluated in the KNHANES. Using the results of pre-bronchodilator only spirometry for assessing COPD may overestimate the number of COPD patients. So, we used "airflow obstruction" instead of COPD. This study also involved relatively healthy patients (because all participants who admitted to the hospital or living in nursing home were not included in KNHANES VI) with a mild airflow obstruction rather than those with a severe airflow obstruction. Finally, the extent of muscle dysfunction in COPD patients is greater in the lower limbs than the upper limbs or the hands. We evaluated only HGS.

Despite these limitations, the strengths of this study include the use of a Korean national-based sample, extensive data regarding potential confounders, and a large sample size that allowed for increased precision and multiple statistical adjustments.

Our study suggests that measuring HGS is a simple, 
inexpensive risk-stratifying method to detect airway obstruction and that HGS might serve as a first test to identify patients with poor upper limb strength who could then undergo more extensive exercise capacity testing. However, pulmonary function test is also a simple, inexpensive, non-invasive test. There is no need to use HGS instead of pulmonary function test. But in developing county, lung function test is expensive and other huddle may be present in private clinic perform lung function test. In that case, HGS is useful alternative method to detect for lung function decline.

However, we think that this test alone is insufficient to inform patients and physicians about exercise capacity because it does not test large or multiple muscles as do other tests. But, HGS might serve as a first easy test to identify patients with poor upper limb muscle strength. It could serve as a screening test that could then undergo more extensive exercise capacity testing. It would be useful to measure HGS simply to detect airway diseases in clinics without spirometry. Measuring HGS is a relatively simple test not definitely dependent on equipment, time, or effort, which may be used to monitor muscle function among patients with obstructive lung disease. Further welldesigned prospective studies on HGS may give us this association with obstructive lung disease and contribute to identifying the need for evaluating outcomes of pulmonary rehabilitation.

\section{Acknowledgements}

None.

\section{Footnote}

Conflicts of Interest: The authors have no conflicts of interest to declare.

\section{References}

1. Gosselink R, Troosters T, Decramer M. Peripheral muscle weakness contributes to exercise limitation in COPD. Am J Respir Crit Care Med 1996;153:976-80.

2. Bohannon RW. Dynamometer measurements of hand-grip strength predict multiple outcomes. Percept Mot Skills 2001;93:323-8.

3. Bohannon RW. Hand-grip dynamometry predicts future outcomes in aging adults. J Geriatr Phys Ther 2008;31:3-10.
4. Puhan MA, Siebeling L, Zoller M, et al. Simple functional performance tests and mortality in COPD. Eur Respir J 2013;42:956-63.

5. Kovarik M, Joskova V, Patkova A, et al. Hand grip endurance test relates to clinical state and prognosis in COPD patients better than 6-minute walk test distance. Int J Chron Obstruct Pulmon Dis 2017;12:3429-35.

6. Gosselink R, Troosters T, Decramer M. Distribution of muscle weakness in patients with stable chronic obstructive pulmonary disease. J Cardiopulm Rehabil 2000;20:353-60.

7. Eisner MD, Blanc PD, Yelin EH, et al. COPD as a systemic disease: impact on physical functional limitations. Am J Med 2008;121:789-96.

8. Marino DM, Marrara KT, Ike D, et al. Study of peripheral muscle strength and severity indexes in individuals with chronic obstructive pulmonary disease. Physiother Res Int 2010;15:135-43.

9. Jeong M, Kang HK, Song P, et al. Hand grip strength in patients with chronic obstructive pulmonary disease. Int $\mathrm{J}$ Chron Obstruct Pulmon Dis 2017;12:2385-90.

10. Lee SH, Kim SJ, Han Y, et al. Hand grip strength and chronic obstructive pulmonary disease in Korea: an analysis in KNHANES VI. Int J Chron Obstruct Pulmon Dis 2017;12:2313-21.

11. US Centers for Disease Control and Prevention (2010). Health behaviors of adults: United States, 2005-2007. Vital and Health Statistics, Series 10, Number 245, Appendix II, p. 80., .

12. Coups EJ, Ostroff JS. A population-based estimate of the prevalence of behavioral risk factors among adult cancer survivors and noncancer controls. Prev Med 2005;40:702-11.

13. Haskell WL, Lee IM, Pate RR, et al. Physical activity and public health: updated recommendation for adults from the American College of Sports Medicine and the American Heart Association. Med Sci Sports Exerc 2007;39:1423-34.

14. Sun Y, Roth DL, Ritchie CS, et al. Reliability and predictive validity of energy intake measures from the 24hour dietary recalls of homebound older adults. J Am Diet Assoc 2010;110:773-8.

15. Ranu H, Wilde M, Madden B. Pulmonary function tests. Ulster Med J 2011;80:84-90.

16. Park HJ, Leem AY, Lee SH, et al. Comorbidities in obstructive lung disease in Korea: data from the fourth and fifth Korean National Health and Nutrition Examination Survey. Int J Chron Obstruct Pulmon Dis 2015;10:1571-82.

17. Roberts HC, Denison HJ, Martin HJ, et al. A review 
of the measurement of grip strength in clinical and epidemiological studies: towards a standardised approach. Age Ageing 2011;40:423-9.

18. Yoo JI, Choi H. Mean Hand grip Strength and Cut-off Value for Sarcopenia in Korean Adults Using KNHANES VI. J Korean Med Sci 2017;32:868-72.

19. Ling CH, Taekema D, de Craen AJ, et al. Hand grip strength and mortality in the oldest old population: the Leiden 85-plus study. Cmaj 2010;182:429-35.

20. Stenholm S, Rantanen T, Heliovaara M, et al. The mediating role of $\mathrm{C}$-reactive protein and hand grip strength between obesity and walking limitation. J Am Geriatr Soc 2008;56:462-9.

21. Leong DP, Teo KK, Rangarajan S, et al. Prognostic value of grip strength: findings from the Prospective Urban Rural Epidemiology (PURE) study. Lancet 2015;386:266-73.

22. Hallin R, Janson C, Arnardottir RH, et al. Relation between physical capacity, nutritional status and systemic inflammation in COPD. Clin Respir J 2011;5:136-42.

23. Strandkvist VJ, Backman H, Roding J, et al. Hand grip strength is associated with forced expiratory volume in 1 second among subjects with COPD: report from a population-based cohort study. Int J Chron Obstruct Pulmon Dis 2016;11:2527-34.

24. Kutsuzawa T, Shioya S, Kurita D, et al. 31P-NMR study of skeletal muscle metabolism in patients with chronic respiratory impairment. Am Rev Respir Dis 1992;146:1019-24.

25. Kutsuzawa T, Shioya S, Kurita D, et al. Muscle energy

Cite this article as: Han CH, Chung JH. Association between hand grip strength and spirometric parameters: Korean National health and Nutrition Examination Survey (KNHANES). J Thorac Dis 2018;10(11):6002-6009. doi: 10.21037/jtd.2018.10.09 metabolism and nutritional status in patients with chronic obstructive pulmonary disease. A 31P magnetic resonance study. Am J Respir Crit Care Med 1995;152:647-52.

26. Bernard S, LeBlanc $P$, Whittom F, et al. Peripheral muscle weakness in patients with chronic obstructive pulmonary disease. Am J Respir Crit Care Med 1998;158:629-34.

27. Hornby ST, Nunes QM, Hillman TE, et al. Relationships between structural and functional measures of nutritional status in a normally nourished population. Clin Nutr 2005;24:421-6.

28. Hegewald MJ, Lefor MJ, Jensen RL, et al. Peak expiratory flow is not a quality indicator for spirometry: peak expiratory flow variability and FEV1 are poorly correlated in an elderly population. Chest 2007;131:1494-9.

29. Fragoso CA, Gahbauer EA, Van Ness PH, et al. Peak expiratory flow as a predictor of subsequent disability and death in community-living older persons. J Am Geriatr Soc 2008;56:1014-20.

30. Sillanpää E, Stenroth L, Bijlsma AY, et al. Associations between muscle strength, spirometric pulmonary function and mobility in healthy older adults. Age (Dordr) 2014;36:9667.

31. Cruz-Jentoft AJ, Baeyens JP, Bauer JM, et al. Sarcopenia: European consensus on definition and diagnosis: Report of the European Working Group on Sarcopenia in Older People. Age Ageing 2010;39:412-23.

32. Buchman AS, Boyle PA, Wilson RS, et al. Respiratory muscle strength predicts decline in mobility in older persons. Neuroepidemiology 2008;31:174-80. 